Preprint typeset in JHEP style - HYPER VERSION

\title{
Boost-invariant early time dynamics from AdS/CFT
}

\author{
Guillaume Beuf ${ }^{a}$, Michal P. Heller ${ }^{b}$, Romuald A. Janik ${ }^{b}$ and Robi Peschanski ${ }^{a *}$ \\ ${ }^{a}$ Institut de Physique Théorique URA 2306, Unité de Recherche associée au CNRS, \\ CEA-Saclay, F-91191 Gif/Yvette Cedex, France. \\ ${ }^{b}$ M.Smoluchowski Institute of Physics, Jagellonian University, \\ Reymonta 4, 30-059 Krakow, Poland.
}

\begin{abstract}
Boost-invariant dynamics of a strongly-coupled conformal plasma is studied in the regime of early proper-time using the AdS/CFT correspondence. It is shown, in contrast with the late-time expansion, that a scaling solution does not exist. The boundary dynamics in this regime depends on initial conditions encoded in the bulk behavior of a Fefferman-Graham metric coefficient at initial proper-time. The relation between the early-time expansion of the energy density and initial conditions in the bulk of AdS is provided. As a general result it is proven that a singularity of some metric coefficient in Fefferman-Graham frame exists at all times. Requiring that this singularity at $\tau=0$ is a mere coordinate singularity without the curvature blow-up gives constraints on the possible boundary dynamics. Using a simple Pade resummation for solutions satisfying the regularity constraint, the features of a transition to local equilibrium, and thus to the hydrodynamical late-time regime, have been observed. The impact of this study on the problem of thermalization is discussed.
\end{abstract}

KEYWORDs: Gauge-gravity correspondence, Heavy Ions.

*guillaume.beuf@cea.fr, michal.heller@uj.edu.pl, ufrjanik@th.if.uj.edu.pl, robi.peschanski@cea.fr 


\section{Contents}

1. Introduction

2. Boost-invariant dynamics and holography

2.1 Energy-momentum tensor of boost-invariant flow

2.2 Holographic reconstruction of space-time 5

3. Late time dynamics 6

3.1 Motivation 6

3.2 The asymptotic solution

3.3 Large proper-time expansion and hydrodynamics 8

4. Early time dynamics 10

4.1 No scaling at early time 10

4.2 The Fefferman-Graham metric is singular at all times 12

4.3 Constraints at early time 14

4.4 Early time expansion of the energy density 16

5. Transition to the hydrodynamic regime 17

5.1 Resummation scheme for the energy density 17

5.2 Qualitative features of the approach to local equilibrium

6. Summary 20

\section{Introduction}

Recently, a new theoretical approach to heavy-ion reactions and the formation of quarkgluon plasma (QGP) in RHIC experiments using the AdS/CFT correspondence arose as a fruitful application of string theory to the real world. The AdS/CFT correspondence allows to describe many properties of a class of gauge theories at strong coupling in terms of a dual gravitational description. What makes it a unique tool is firstly that it works well in Minkowski signature allowing for a description of inherently time-dependent phenomena, in contrast to lattice QCD methods which are directly tied to Euclidean signature. Secondly, explicit analytical computations are often possible and one can get new insight into strongly coupled dynamics from the dual geometric perspective.

On the experimental side, the features of the distribution of particles observed in the final stage of the reaction point to the existence of a hydrodynamical regime of the QGP expansion [1, 2], in particular the observed elliptic flow denoting sizeable collective effects 


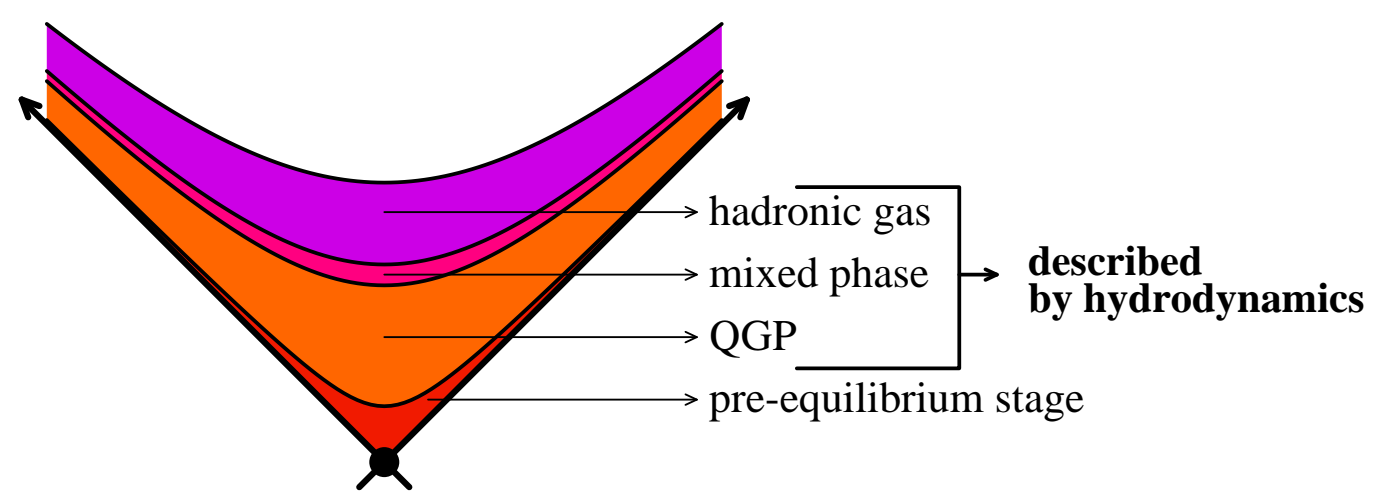

Figure 1: Description of QGP formation in heavy ion collisions. The kinematic landscape is defined by $\tau=\sqrt{\left(x^{0}\right)^{2}-\left(x^{3}\right)^{2}} ; y=\frac{1}{2} \log \frac{x^{0}+x^{3}}{x^{0}-x^{3}} ; x_{\perp}=\left\{x^{1}, x^{2}\right\}$, where the coordinates along the light-cone are $x^{0} \pm x^{1}$, the transverse ones are $\left\{x^{1}, x^{2}\right\}$ and $\tau$ is the proper time, $y$ the "space-time rapidity".

[3]. The hydrodynamic regime has to last long enough and start soon enough after the collision in order to explain the observed collective effects. Moreover, the smallness of the viscosity which can be extracted from hydrodynamical simulations describing the data leads to an almost-perfect fluid behaviour of the QGP, and thus to a short mean-free path inside the fluid. Putting together these experimental inputs, and in order to go beyond a mererly phenomenological description, it appears to be theoretically necessary to investigate as much as possible the properties of a strongly-coupled Quantum-Chromodynamic plasma.

In the absence of nonperturbative methods applicable to real-time dynamics of strongly coupled Quantum Chromodynamic (QCD) plasma, one is led to consider similar problems from the point-of-view of the AdS/CFT correspondence, that is looking for the characteristics of plasma in a gauge theory for which the AdS/CFT correspondence takes its simplest form - the $\mathcal{N}=4$ supersymmetric Yang-Mills theory [4] which possesses a known and tractable gravity dual.

Although the $\mathcal{N}=4$ gauge theory is supersymmetric and conformal and thus quite different from QCD at zero temperature, both supersymmetry and scale-invariance are broken explicitly at finite temperature and we may expect qualitative similarities with QCD plasma for a range of temperatures above the QCD deconfinement phase transition ${ }^{1}$.

Indeed, the gauge/gravity dual calculation [5] showing, in a static setting, that the viscosity over entropy ratio $\eta / s$ is very small (equal to $1 / 4 \pi$ ) and even suggesting a universal lower bound, is in qualitative agreement with hydrodynamic simulations of QCD plasma and was a poweful incentive to explore further the AdS/CFT duality approach.

In order to go beyond static calculations, one has to adapt the dual AdS/CFT approach to the relativistic kinematic framework of heavy-ion reactions, where two ultra-relativistic heavy nuclei collide and form an expanding medium, see Fig.11. It is convenient, initially,

\footnotetext{
${ }^{1}$ There exist more refined versions of the AdS/CFT correspondence which may have more features in common with QCD, however the gravity backgrounds are much more complicated and we will not consider them here.
} 
to adopt the simplest setting in which the relevant physics is still present.

For this sake, one is guided by the seminal work of Bjorken [6], who made a simplifying assumption of boost-invariant dynamics for his hydrodynamic description of high-energy heavy-ion collisions. Hence, the different stages of the plasma formation, expansion and hadronization, see Fig. 1, depend only on the proper-time $\tau$ and not on rapidity ${ }^{2} y$. This assumption is indeed phenomenologically valid for the central rapidity region of the collision at high energy.

For the application of the AdS/CFT correspondence to this setting, and in particular for finding the gravity dual [7], the above symmetry assumptions allow for a drastic simplification of an otherwise yet probably intractable problem, since the dual dynamics depends only on two variables, $\tau$, proper-time and $z$, the bulk variable in the dual asymptotically AdS spacetime.

The two main physical questions ${ }^{3}$ are to understand the behaviour of quark gluon plasma at late and early times. At late times, we would like to derive the appearance of hydrodynamic behaviour with all its characteristics, asymptotically perfect fluid flow, low viscosity, transport coefficients, etc. At early times, we would like to understand the rapid thermalization starting from the ultra-relativistic initial conditions.

In fact the study of the former problem has led to quite successful developments, showing that the hydrodynamic boost-invariant Bjorken flow is a consequence of late-time dynamics of gravity in the dual side of the AdS/CFT correspondence. Subsequently the way in which hydrodynamics arises from the AdS/CFT has been understood even without using any symmetry arguments [8] as a gradient expansion of Einstein's equations. However since our main interest in this paper is the radically different early time regime where such expansions are inapplicable, we will use the full system of Einstein's equations with the boost-invariant symmetry assumptions. Before that, we will first recall the analysis for late times, since the point of departure will be analogous for the early time problem, but with different developments and results.

The second problem, which forms the novel part of our investigations in the present study, is to go beyond hydrodynamics and investigate early-time dynamics. The only assumption we will make, apart from boost-invariance, is the existence of a strongly coupled pre-hydrodynamic stage ${ }^{4}$ allowing for the use of the AdS/CFT correspondence in the same framework as for late times, and thus to look for a connection of the two regimes in a unified scheme. In particular, one is curious to understand the transition towards the hydrodynamic regime and thus to get some information about the puzzling problem of the apparently short [2] thermalization time.

Summarizing the contents of the paper, the next section recalls the basic AdS/CFT approach to boost-invariant dynamics. The main results of the late-time solution are

\footnotetext{
${ }^{2}$ For the Bjorken flow, spatial and momentum rapidity are equal.

${ }^{3}$ We do not address here another important problem which is hadronization, since it requires to go beyond our current understanding of the presently known Gauge/Gravity dualities.

${ }^{4}$ Physically, it may be that the initial stage of the collision has some weak-coupling properties, for instance in the Color Glass Condensate (CGC) setting [9], but we will not consider here this possibility (for a recent attempt to describe CGC initial conditions within the AdS/CFT see [10]).
} 
given in section 3, with emphasis on those aspects which will be useful for the early-time regime. Section 4 is devoted to the theoretical analysis of the early-time boost-invariant dual dynamics, while section 5 deals with numerical solutions with the goal of studying the transition to hydrodynamics. Conclusions and outlook are given in the final section.

\section{Boost-invariant dynamics and holography}

\subsection{Energy-momentum tensor of boost-invariant flow}

Currently the best understanding of non-perturbative non-equillibrium 4-dimensional quantum field theories is provided by string theory methods. Holography translates gauge theory problems in four dimensions at large number of colors and strong ('t Hooft) coupling into higher-dimensional classical gravity. Einstein's equations involve then, in the simplest setting, functions of both gauge theory dimensions and of one more variable $z$ parameterizing the radial direction in AdS. In a general setup, finding solutions of Einstein's equations must involve numerical analysis and is rather difficult. Moreover, singular components of the metric may and indeed do appear [7, 11, 12] which are not easy to implement numerically, unless their structure is known beforehand. It is thus important to focus on the simplest possible, yet phenomenologically interesting, gauge theory dynamical processes. As explained in the introduction, we will focus on the boost-invariant plasma expansion. This setup serves as a first approximation of dynamical plasma created at RHIC, as excited nuclear matter undergoes one-dimensional expansion and is both translationally and rotationally invariant in the plane perpendicular to the expansion axis (physical quantities do not depend on $x^{1}, x^{2}$ ). The crucial simplifying assumption is boost-invariance along the expansion axis, which can be most easily imposed when passing to proper-time $(\tau)$ and (spatial) rapidity $(y)$ coordinates related to the usual lab-frame time $\left(x^{0}\right)$ and position along the expansion axis $\left(x^{3}\right)$ by

$$
\begin{aligned}
& x^{0}=\tau \cosh y \\
& x^{3}=\tau \sinh y .
\end{aligned}
$$

Under a boost along the $x^{3}$ direction proper-time does not change, whereas rapidity is shifted by a constant. Thus in this coordinate frame boost-invariance means that physical observables do not depend on rapidity.

A quantity of special interest is the expectation value of the energy-momentum tensor of the plasma. In the $\left(\tau, y, x^{1}, x^{2}\right)$ frame with all mentioned symmetries ${ }^{5}$ imposed, the most general boost-invariant energy-momentum tensor takes the form

$$
T_{\mu \nu}=\operatorname{diag}\left\{\epsilon(\tau), \tau^{2} p_{\|}(\tau), p_{\perp}(\tau), p_{\perp}(\tau)\right\} .
$$

Imposing energy-momentum conservation $\nabla_{\mu} T^{\mu \nu}=0$ (in boost-invariant case there is only one independent equation) and tracelessness $T_{\mu}^{\mu}=0$ leads to the energy momentum tensor fully specified in terms of a single function of one variable - the energy density $\epsilon(\tau)$, namely

\footnotetext{
${ }^{5}$ Together with parity invariance in rapidity, which ensures that there are no nondiagonal $T_{\tau y}$ terms in the energy-momentum tensor.
} 


$$
T_{\mu \nu}=\operatorname{diag}\left\{\epsilon(\tau),-\tau^{2}\left(\epsilon(\tau)+\tau \epsilon^{\prime}(\tau)\right), \epsilon(\tau)+\frac{1}{2} \tau \epsilon^{\prime}(\tau), \epsilon(\tau)+\frac{1}{2} \tau \epsilon^{\prime}(\tau)\right\}
$$

The function $\epsilon(\tau)$ may be arbitrary at this level and it is the gravitational dual which will single out the allowed ones. Note that $T_{\mu \nu}$ is submitted to an important positivity constraint, namely $T_{\mu \nu} t^{\mu} t^{\nu} \geq 0$, for any time-like vector $t^{\mu}$ implying []]

$$
-\frac{4 \epsilon(\tau)}{\tau} \leq \epsilon^{\prime}(\tau) \leq 0
$$

This requirement may perhaps be lifted in case of some highly quantum states [13 appearing at transient times, but we expect that it should hold for any more macroscopic configuration. We observe a temporary violation of this constraint for a specific initial condition (see section 5).

\subsection{Holographic reconstruction of space-time}

The AdS/CFT correspondence maps the expectation value of the energy-momentum tensor operator into the behavior of the 5-dimensional asymptotically AdS metric $G_{A B}$. This metric is a solution of Einstein's equations with negative cosmological constant $\Lambda=-6 L^{-2}$ (where $\mathrm{L}$ is the AdS radius ${ }^{6}$ ), namely

$$
R_{A B}-\frac{1}{2} R \cdot G_{A B}-6 G_{A B}=0 .
$$

The general asymptotically AdS metric written in Fefferman - Graham coordinates [14 takes the form

$$
\mathrm{d} s^{2}=G_{A B} \mathrm{~d} y^{A} \mathrm{~d} y^{B}=\frac{g_{\mu \nu} \mathrm{d} x^{\mu} \mathrm{d} x^{\nu}+\mathrm{d} z^{2}}{z^{2}}
$$

where $z$ is the AdS radial variable and $g_{\mu \nu}$ is a four-dimensional metric on constant $z$ slices, which depends both on the field theory directions $x^{\mu}$ and $z$. The hypersurface at $z=0$ is a conformal boundary of AdS and is identified with the metric in which the CFT lives. In the considered field theory setup this is the 4-dimensional Minkowski metric $\eta_{\mu \nu}$. Einstein's equations obtained from (2.5)) (see further Eq. (4.10) for the full set of Einstein's equations expressed in Fefferman-Graham coordinates) can be solved pertubatively in the vicinity of the conformal theory. The four-dimensional part $g_{\mu \nu}$ of the AdS metric ${ }^{7}$

$$
g_{\mu \nu}=g_{\mu \nu}^{(0)}+g_{\mu \nu}^{(2)} z^{2}+g_{\mu \nu}^{(4)} z^{4}+g_{\mu \nu}^{(6)} z^{6}++g_{\mu \nu}^{(8)} z^{8}+\ldots
$$

can be obtained order by order in $z^{2}$ from the equations of motion (2.5). The leading piece in (2.7) is the Minkowski metric $\eta_{\mu \nu}$, which in the proper $\left(\tau, y, \mathbf{x}_{\perp}\right)$ variables takes the form

\footnotetext{
${ }^{6}$ In the rest of the paper the AdS radius $\mathrm{L}$ will be set to 1 , but can be trivially restored on dimensional grounds. Greek and latin indices denote respectively field theory and bulk AdS directions.

${ }^{7}$ All the formulas regarding holographic reconstruction of the bulk space time are provided for the case when the boundary is the Minkowski space.
} 


$$
g_{\mu \nu}^{(0)} \mathrm{d} x^{\mu} \mathrm{d} x^{\nu}=\eta_{\mu \nu} \mathrm{d} x^{\mu} \mathrm{d} x^{\nu}=-\mathrm{d} \tau^{2}+\tau^{2} \mathrm{~d} y^{2}+\mathrm{d} \mathbf{x}_{\perp}^{2}
$$

The quadratic term $g_{\mu \nu}^{(2)}$ vanishes identically, and the first non-trivial term $g_{\mu \nu}^{(4)}$ turns out to be proportional to the expectation value of the energy-momentum tensor [15]

$$
g_{\mu \nu}^{(4)}=\frac{2 \pi^{2}}{N_{c}^{2}}\left\langle T_{\mu \nu}\right\rangle
$$

Higher order contributions $\left(g_{\mu \nu}^{(2 n)}\right.$ for $\left.n>2\right)$ can be obtained from the Einstein's equations and are fully expressed in terms of $\left\langle T_{\mu \nu}\right\rangle$ and its derivatives. At this level there are no constraints on the form of the energy-momentum tensor - any conserved and traceless one will do (apart from the positivity conditions (2.4)).

However it is to be expected [7] that generic energy-momentum tensor would lead to singularities in the bulk of AdS, which are beyond the scope of a perturbative solution in $z^{2}$. Therefore in order to have a control over the geometry in the region where potential singularities may develop, one needs to solve the Einstein's equations deeper in the bulk. Since the energy-momentum tensor for the boost-invariant flow takes a particularly simple diagonal form (2.2), in this case we are led to adopt the 5-dimensional Fefferman-Graham metric ansatz satisfying the symmetries of boundary dynamics

$$
\mathrm{d} s^{2}=\frac{-e^{a(\tau, z)} \mathrm{d} \tau^{2}+\tau^{2} e^{b(\tau, z)} \mathrm{d} y^{2}+e^{c(\tau, z)} \mathrm{d} \mathbf{x}_{\perp}^{2}+\mathrm{d} z^{2}}{z^{2}} .
$$

The full set of Einstein's equations corresponding to the parametrized metric (2.10) are given in (4.10). Solving the Einstein's equations for any value of $\tau$ and $z$ is very difficult and must probably involve numerical procedures. However it is possible to gain nevertheless some analytical insight. There are two regimes where some simplifications may occur these are for small or large values of $\tau$. In both cases there is a small parameter (either $\tau$ or its inverse), which can be used to construct the geometry in perturbative manner in $\tau$, but exactly in $z$. So we shall focus on late and early time dynamics.

\section{Late time dynamics}

\subsection{Motivation}

At sufficiently late times, the boost-invariant plasma is expected to be locally equilibrated and thus well-described by hydrodynamics. However, it is a priori not obvious how to prove it on the gravity side of the AdS/CFT. The solution was first shown in [7] and then further developed in [16, 11, 17, 12, 18, 19, 20, 21] (see [22] for a review). The following subsections review how to obtain the late-time boost-invariant dynamics perturbatively, since the methods developed for this approach will be crucial in the rest of the paper when

considering the open problem of early time dynamics and the corresponding transition to local equilibrium. 


\subsection{The asymptotic solution}

The goal of the whole approach both in the early and late times domain is to solve the Einstein's equations (4.10) perturbatively in proper time $\tau$, but (at each order in $\tau$ ) exactly in the radial AdS variable $z$. This can be achieved by resumming the power series in $z^{2}$ for the bulk metric (2.7). For arbitrary proper-time dependence of the energy density it is yet out of reach, but focusing on the large proper time regime and assuming that the energy density of the boost-invariant plasma exhibits a power-like tail

$$
\epsilon(\tau) \sim \frac{\#}{\tau^{s}}+\ldots
$$

for some positive power ${ }^{8}$ of $s$ this can be basically done. The crucial observation of Ref. [7] is the existence of a scaling variable $v=z \tau^{-s / 4}$, which captures the large proper-time behavior on the gravity side (see section (4.1) for more details). Taking the $\tau \rightarrow \infty$ limit while keeping the scaling variable fixed reduces the Einstein's equations (4.10) for the functions $a(\tau, z), b(\tau, z)$ and $c(\tau, z)$ to ordinary nonlinear differential equations for their scaling forms $a_{0}(v), b_{0}(v)$ and $c_{0}(v)$, which can be solved exactly leading to

$$
\begin{aligned}
& a_{0}(v)=A(v)-2 m(v), \\
& b_{0}(v)=A(v)+(2 s-2) m(v), \\
& c_{0}(v)=A(v)+(2-s) m(v),
\end{aligned}
$$

where

$$
\begin{aligned}
& A(v)=\frac{1}{2}\left\{\log \left(1+\Delta(s) v^{4}\right)+\log \left(1-\Delta(s) v^{4}\right)\right\}, \\
& m(v)=\frac{1}{4 \Delta(s)}\left\{\log \left(1+\Delta(s) v^{4}\right)-\log \left(1-\Delta(s) v^{4}\right)\right\}
\end{aligned}
$$

with

$$
\Delta(s)=\sqrt{\frac{3 s^{2}-8 s+8}{24}} .
$$

It turns out however, that the geometry is generically singular for all the values of $s$, apart from $s=4 / 3$. This can be checked by evaluating the square of the Riemann tensor (i.e. Kretschmann scalar) in the scaling limit and requiring its regularity (the authors of Ref. 23. made also the observation that $s=4 / 3$ is the only value for which the functions (3.2) are single-valued).

For $s=4 / 3$ the energy-momentum tensor takes asymptotically the form

\footnotetext{
${ }^{8}$ The power $s$ is constrained to take values in the range $0 \leq s<4$ assuming positivity of the energy density (2.4) in any time-like reference frame. There are three particular phenomenologically interesting values of $s$ within this range: $s=4 / 3, s=1$ or $s=0$. The first one leads to the perfect fluid case where $p_{\perp}(\tau)=p_{\|}(\tau)$ and the second one to the free streaming scenario with $p_{\|}(\tau)=0$. The third interesting regime discussed in the literature [23], is the limiting case $s=0$ with $p_{\perp}(\tau)=-p_{\|}(\tau)$ which will be adressed in section 5 .
} 


$$
T_{\mu \nu}=\operatorname{diag}\left\{\frac{\#}{\tau^{4 / 3}}+\ldots, \tau^{2} \frac{1}{3} \frac{\#}{\tau^{4 / 3}}+\ldots, \frac{1}{3} \frac{\#}{\tau^{4 / 3}}+\ldots, \frac{1}{3} \frac{\#}{\tau^{4 / 3}}+\ldots\right\}
$$

where \# denotes some numerical constant. From this expression it is clear that $p_{\|}(\tau)=$ $p_{\perp}(\tau)$ (asymptotically), the system is in local equilibrium and is described by the perfect fluid hydrodynamics. The gravity dual in this scaling limit is given then by

$\mathrm{d} s_{\tau \rightarrow \infty}^{2}=-\frac{\left(1-z^{4} \cdot \tau^{-4 / 3}\right)^{2}}{z^{2}\left(1+z^{4} \cdot \tau^{-4 / 3}\right)} \mathrm{d} \tau^{2}+\frac{1}{z^{2}} \tau^{2}\left(1+z^{4} \cdot \tau^{-4 / 3}\right) \mathrm{d} y^{2}+\frac{1}{z^{2}}\left(1+z^{4} \cdot \tau^{-4 / 3}\right) \mathrm{d} \mathbf{x}_{\perp}^{2}+\frac{1}{z^{2}} \mathrm{~d} z^{2}$

and looks like a boosted and dilated black brane

$$
\mathrm{d} s^{2}=-\frac{\left(1-z^{4} \lambda^{4}\right)^{2}}{z^{2}\left(1+z^{4} \lambda^{4}\right)} u_{\mu} u_{\nu} \mathrm{d} x^{\mu} \mathrm{d} x^{\nu}+\frac{1}{z^{2}}\left(1+z^{4} \lambda^{4}\right)\left(\eta_{\mu \nu}+u_{\mu} u_{\nu}\right) \mathrm{d} x^{\mu} \mathrm{d} x^{\nu}+\frac{1}{z^{2}} \mathrm{~d} z^{2}
$$

with boost parameter $u^{\mu}=1 \cdot\left[\partial_{\tau}\right]^{\mu}$ and dilatation $\lambda \sim \tau^{-1 / 3}$. Recently this connection has been exploited and formulated as a fluid/gravity duality [8]. Note that (3.6) contains a $(\tau \tau)$ coefficient which goes to zero at $z \sim \tau^{1 / 3}$, giving a hint for a black brane horizon [24].

It is also clear that the metric (3.6) is not an exact solution of Einstein's equations there are subleading effects not captured by the scaling variable limit. Those with powerlike scaling correspond to dissipative corrections in hydrodynamics.

\subsection{Large proper-time expansion and hydrodynamics}

When evaluating $\mathcal{R}^{2} \equiv \mathcal{R}_{A B C D} \mathcal{R}^{A B C D}$ in the scaling limit $\left(\tau \rightarrow \infty\right.$ keeping $v=z \cdot \tau^{-1 / 3}$ fixed) on the asymptotic solution, the following pattern is encountered

$$
\mathcal{R}^{2}=(\text { nonsingular }) \mathcal{R}_{(0)}^{2}(v)+\frac{1}{\tau^{4 / 3}} \cdot(\text { singular }) \mathcal{R}_{(4 / 3)}^{2}(v)+\ldots
$$

where (singular) $\mathcal{R}_{(4 / 3)}^{2}(v)$ has a fourth-order pole at $v=3^{1 / 4}$ and the dots "..." denote terms suppressed by inverse powers of $\tau$ higher than 4/3. Any background with singularities not covered by the event horizon would correspond to unphysical configurations on the gauge theory side (see e.g. [25, 26]). Thus the condition of nonsingularity is the crucial requirement, which singles out the correct behavior of the geometry and thus of the energymomentum tensor of the boundary plasma. It is obvious that the singularity at $\tau^{-4 / 3}$ in (3.8) can be cancelled only if there is an additional contribution to the metric warp factors which arises from a correction to the perfect fluid asymptotics

$$
\varepsilon(\tau)=\frac{1}{\tau^{\frac{4}{3}}}\left(1+\frac{\#}{\tau^{r}}+\ldots\right)
$$

Assuming now the expansion

$$
a(\tau, z)=a_{0}\left(z \cdot \tau^{-1 / 3}\right)+\frac{1}{\tau^{r}} a_{1}\left(z \cdot \tau^{-1 / 3}\right)+\frac{1}{\tau^{4 / 3}} a_{2}\left(z \cdot \tau^{-1 / 3}\right)+\ldots
$$


with analogous equations for the other warp factors $b$ and $c$ and solving Einstein's equations at orders $\tau^{-r}$ and $\tau^{-4 / 3}$ for $r \neq 2 / 3$ one ends up with a singular Kretschmann scalar $\mathcal{R}^{2}$ of the following form

$$
\begin{aligned}
\mathcal{R}^{2}= & \text { (nonsingular }) \mathcal{R}_{(0)}^{2}(v)+\frac{1}{\tau^{r}} \cdot(\text { nonsingular }) \mathcal{R}_{(r)}^{2}(v)+ \\
& +\frac{1}{\tau^{4 / 3}} \cdot(\text { singular }) \mathcal{R}_{(4 / 3)}^{2}(v)+\frac{1}{\tau^{2 r}} \cdot(\text { singular }) \mathcal{R}_{(2 r)}^{2}(v)
\end{aligned}
$$

However for $r=2 / 3$ the singular contribution at $\tau^{-2 r}$ cancels the singularities at $\tau^{-4 / 3}$ (see 12 for details) and gives a regular Kretschmann scalar up to the terms of order $\tau^{-4 / 3}$ in the scaling limit. Physically, on the gauge theory side, this exactly corresponds to the first viscous corrections to the perfect fluid expansion. This argument repeated at any higher order fixes the large proper-time expansion of the bulk metric to take the form

$$
\begin{aligned}
& a(\tau, z)=a_{0}\left(z \tau^{-1 / 3}\right)+\frac{1}{\tau^{2 / 3}} a_{1}\left(z \tau^{-1 / 3}\right)+\frac{1}{\tau^{4 / 3}} a_{2}\left(z \tau^{-1 / 3}\right)+\ldots \\
& b(\tau, z)=b_{0}\left(z \tau^{-1 / 3}\right)+\frac{1}{\tau^{2 / 3}} b_{1}\left(z \tau^{-1 / 3}\right)+\frac{1}{\tau^{4 / 3}} b_{2}\left(z \tau^{-1 / 3}\right)+\ldots \\
& c(\tau, z)=c_{0}\left(z \tau^{-1 / 3}\right)+\frac{1}{\tau^{2 / 3}} c_{1}\left(z \tau^{-1 / 3}\right)+\frac{1}{\tau^{4 / 3}} c_{2}\left(z \tau^{-1 / 3}\right)+\ldots
\end{aligned}
$$

The functions $a_{i}(v), b_{i}(v)$ and $c_{i}(v)$ can be obtained order by order solving Einstein's equations in the scaling variable. At each order $i>0$ there is a single integration constant fixed neither by the equations of motion nor the asymptotic behavior of the metric, which is a combination of various transport coefficients of the plasma. Each constant ${ }^{9}$ in the expansion of the energy density or equivalently the temperature $\left(\epsilon \sim T^{4}\right)$

$$
T(\tau)=\frac{\Lambda}{\tau^{1 / 3}}\left\{1-\frac{1}{\Lambda \tau^{2 / 3}} \cdot \frac{\eta_{0}}{\sqrt{2} 3^{1 / 4} \pi}+\frac{1}{\Lambda^{2} \tau^{4 / 3}}\left(\frac{\lambda_{1}^{(0)}}{3 \sqrt{3} \pi^{2}}-\frac{\eta_{0} \tau_{\Pi}^{(0)}}{3 \sqrt{3} \pi^{2}}\right)+\ldots\right\}
$$

is fixed uniquely by requiring the nonsingularity of the geometry at one order higher. In the above equation $\Lambda$ denotes the overall energy scale defined by $T=\Lambda \cdot \tau^{-1 / 3}+\ldots, \eta_{0}$ comes from the shear viscosity, while $\lambda_{1}^{(0)}$ and $\tau_{\Pi}^{(0)}$ are related to second order transport coefficients $\lambda_{1}$ and relaxation time $\tau_{\Pi}$ (see [27]). The expansion in $\tau^{-2 / 3}$ can be easily understood using hydrodynamics (see e.g. [8] for a detailed discussion), which is an effective description of systems at local equilibrium described by four degrees of freedom: the four-velocity $u^{\mu}$ $\left(u_{\mu} u^{\mu}=-1\right)$ and the temperature $T$. Those quantities vary over space-time, however the scale set by their variations is much bigger than the microscopic scale given by the temperature. Thus the small parameter in this case is $T^{-1} \nabla_{\mu} u^{\nu}$. In a boost-invariant setup the asymptotic scaling of the temperature obtained from the nonsingularity is $\tau^{-1 / 3}$, the covariant derivative of the velocity ${ }^{10}$ scales as $\tau^{-1}$ which leads to the expansion parameter

\footnotetext{
${ }^{9}$ The constant $\eta_{0}$ in first order, $\frac{\lambda_{1}^{(0)}}{3 \sqrt{3} \pi^{2}}-\frac{\eta_{0} \tau_{\Pi}^{(0)}}{3 \sqrt{3} \pi^{2}}$ in the second order, ...

${ }^{10}$ In a boost-invariant setup the fluid velocity takes the form $u^{\mu}=1 \cdot\left[\partial_{\tau}\right]^{\mu}$, but the metric in $\tau-y$ coordinates has nonvanishing Christoffel symbols leading to $\nabla u=\tau^{-1}$.
} 
$\tau^{-2 / 3}$ in exact agreement with the one obtained on the gravity side. For phenomenological reasons (from causality, see the discussion in e.g. [27]) it is interesting to focus on an energy density expanded up to the second order in $\tau^{-2 / 3}$ expansion, so that the geometry has to be solved up to the third order to fix all the constants of interest.

In 12] a surprising feature showed up: the pole contributions to the Kretschmann scalar at the third order cancelled, leaving a logarithmic singularity of the form $\log \left(3^{1 / 4}-v\right)$. This singularity was present also in higher order curvature invariants in Fefferman-Graham variables, such as $\mathcal{R}_{A B C D} \mathcal{R}^{C D E F} \mathcal{R}_{E F}{ }^{A B}$, which led to the conjecture that the gravity dual to boost-invariant flow cannot be realized within the supergravity approximation [18, 19]. The resolution of this puzzle involves resummation of the series

$$
\mathcal{R}^{2}=\mathcal{R}_{0}^{2}(v)+\frac{1}{\tau^{2 / 3}} \mathcal{R}_{1}^{2}(v)+\frac{1}{\tau^{4 / 3}} \mathcal{R}_{2}^{2}(v)+\frac{1}{\tau^{2}} \mathcal{R}_{3}^{2}(v)+\ldots
$$

which turns out to be equivalent to the change of variables from Fefferman-Graham to Eddington-Finkelstein metric [20]. Subsequently it was shown, that the geometry dual to the boost-invariant flow in the large $\tau$ limit is well-defined at any order of $\tau^{-2 / 3}$ expansion 21] and the usual black brane singularity is covered by an event horizon [24, 28]. Thus the universal behavior of boost-invariant plasma at large proper-time (note that the energy density contains only one arbitrary constant, which sets the overall scale) possesses a regular bulk description, which could be constructed analytically in a perturbative manner. Moreover, as expected, it does not matter which coordinate frame in the bulk is used, so that in the rest of the paper all the calculations will be performed in Fefferman-Graham coordinates.

\section{Early time dynamics}

\subsection{No scaling at early time}

The second regime where analytic methods can be applied is the domain of early times. At first sight it seems that the early-time problem could be addressed by the same method which was so useful for late time, namely solving the boost-invariant Einstein's equations (4.10) without specifying a priori initial conditions. However after careful consideration a crucial difference turns out to occur: no scaling solution appears which would consist of a hierarchy of terms similar to (3.5) and independent of the initial conditions. In fact, on a more physical ground, the initial conditions should play a crucial role at early times. This would indeed agree with the physical intuition which suggests that this regime should not be universal, but rather depend on initial conditions. However, as discussed in the following, the solutions are submitted nevertheless to nontrivial constraints if they are considered in the strong coupling limit.

As will be shown in the rest of the paper the search for a scaling variable (as in Ref. [23]) is invalidated due to a subtlety. In order to see this one needs to recall the way how to identify the existence of a scaling variable. Consider the leading proper time dependence of the energy density in the regime of interest $(\tau \rightarrow \infty$ or $\tau \rightarrow 0)$

$$
\varepsilon(\tau) \sim \frac{1}{\tau^{s}}
$$


and then solve the Einstein's equations (4.10) exactly as a power series around the boundary

$$
g_{\mu \nu}=\eta_{\mu \nu}+g_{\mu \nu}^{(4)}(\tau) z^{4}+g_{\mu \nu}^{(6)}(\tau) z^{6}+g_{\mu \nu}^{(8)}(\tau) z^{8}+\ldots
$$

The coefficients of this power series are explicit $s$-dependent functions of proper time $\tau$ and typically contain a couple of terms. If the part of $g_{\mu \nu}^{(n)}(\tau)$ which dominates at $\tau \rightarrow \infty$ is selected, the above power series becomes a power series of a scaling variable $v=z / \tau^{\frac{s}{4}}$. If on the other hand the parts that dominate for $\tau \rightarrow 0$ are considered, it leads [23] to an expression of the form

$$
\frac{z^{4}}{\tau^{s}} \cdot f\left(w \equiv \frac{z}{\tau}\right)
$$

for the metric coefficients. One consequently finds a unique solution in $w$ for each $s$ with a complex branch cut singularity for $s>0$. This leads to the only possible value $s=0$ right at the margin of the allowed range, i.e.

$$
\varepsilon(\tau) \sim \text { const } \quad \text { for } \quad \tau \rightarrow 0
$$

out of the range of generic $s$ [23].

However the situation when $s=0$ is special. Indeed, going back to the derivation of the early time scaling variable $w=z / \tau$, it turns out that the terms in $g_{\mu \nu}^{(n)}(\tau)$ which lead to it are all multiplied by a factor of $s$. Hence for $s=0$, these terms vanish and a completely different hierarchy of terms appear.

Indeed, if one performs for instance the power series expansion of $a(\tau, z)$ starting from $\epsilon(\tau)=1 / \tau^{s}$ then the answer for the first three orders is

$$
\begin{aligned}
& -z^{4} \tau^{-s}+z^{6}\left\{\frac{1}{6} \tau^{-s-2} s-\frac{1}{12} \tau^{-s-2} s^{2}\right\}+ \\
& +z^{8}\left\{-\frac{1}{16} \tau^{-2 s} s^{2}-\frac{1}{6} \tau^{-2 s}+1 / 6 \tau^{-2 s} s+\frac{1}{96} \tau^{-s-4} s^{2}-\frac{1}{384} \tau^{-s-4} s^{4}\right\}+\ldots .
\end{aligned}
$$

Consider now the term proportional to $z^{8}$ in $(4.5)$. There are two structures $z^{8} / \tau^{(s+4)}$ and $z^{8} / \tau^{2 s}$. The first of these leads to the early-time scaling variable proposed in [23] since it dominates for nonzero $s$. However its coefficient is proportional to $s$ so for $s=0$ it is absent and the only contribution comes from the second term which is not contained in the scaling-variable analysis. The same applies obviously already to the term in $z^{6} / \tau^{(s+2)}$ as well as to all subsequent orders.

Analysing the power series solutions in more detail, one finds that for a generic early time expansion of the energy density ${ }^{11}$

$$
\varepsilon(\tau)=\sum_{n=0}^{\infty} \varepsilon_{2 n} \tau^{2 n},
$$

the coefficients $a_{n}$ of the power series expansion in $z$ of the metric coefficients at $\tau=0$

$$
a(\tau=0, z)=\sum_{n=0}^{\infty} a_{n} z^{4+2 n}
$$

\footnotetext{
${ }^{11}$ The restriction to even powers of $\tau$ is discussed in one of the following sections.
} 
depends on all coefficients $\varepsilon_{2 n}$ in (4.6). Reformulating this observation, this means that each of the possible initial conditions (4.7) leads to a distinct proper-time evolution (4.6), where the coefficients of the two power series are linked through the Einstein's equations. The following mapping is therefore obtained

$$
a(\tau=0, z)=\sum_{n=0}^{\infty} a_{n} z^{4+2 n} \quad \Longrightarrow \quad \varepsilon(\tau)=\sum_{n=0}^{\infty} \varepsilon_{2 n} \tau^{2 n} .
$$

On the other hand, certain nontrivial constraints limit the range of solutions. Among these, the nonsingularity condition on the metric-invariant properties of the geometry plays the crucial role.

The derivation of this result together with the constraints including the nonsingularity argument restricting the early time expansion in terms of even powers of the proper time of the energy density, is the main result of this paper and is presented in section (4.4).

The objective now is to analyze the qualitative properties of the evolution of the energy density starting from the initial conditions (4.7). But before this can be done, the space of allowed initial conditions has to be investigated in more detail, as these have to satisfy a nonlinear constraint equation which is a part of the Einstein's equations (4.10).

\subsection{The Fefferman-Graham metric is singular at all times}

This section focuses on the restrictions on the initial conditions coming from Einstein's equations, which can be written in an equivalent simplified form as

$$
R_{A B}+4 G_{A B}=0
$$

or explicitly in Fefferman-Graham coordinates as

$$
\begin{aligned}
& (\tau \tau): \ddot{b}+2 \ddot{c}-\frac{\dot{a}}{2}(\dot{b}+2 \dot{c})+\frac{1}{2}\left(\dot{b}^{2}+2 \dot{c}^{2}\right)-\frac{1}{\tau}(\dot{a}-2 \dot{b})=e^{a}\left\{a^{\prime \prime}-\frac{3 a^{\prime}}{z}+\left(\frac{a^{\prime}}{2}-\frac{1}{z}\right)\left(a^{\prime}+b^{\prime}+2 c^{\prime}\right)\right\} \\
& (y y): \ddot{b}-\dot{a} \dot{b}+\frac{1}{\tau}(\dot{b}-2 \dot{a})+\frac{1}{2}(\dot{a}+\dot{b}+2 \dot{c})\left(\dot{b}+\frac{2}{\tau}\right)=e^{a}\left\{b^{\prime \prime}-\frac{3 b^{\prime}}{z}+\left(\frac{b^{\prime}}{2}-\frac{1}{z}\right)\left(a^{\prime}+b^{\prime}+2 c^{\prime}\right)\right\}, \\
& (\perp \perp): \ddot{c}-\dot{a} \dot{c}+\frac{\dot{c}}{2}\left(\dot{a}+\dot{b}+2 \dot{c}+\frac{2}{\tau}\right)=e^{a}\left\{c^{\prime \prime}-\frac{3 c^{\prime}}{z}+\left(\frac{c^{\prime}}{2}-\frac{1}{z}\right)\left(a^{\prime}+b^{\prime}+2 c^{\prime}\right)\right\} \\
& (\tau z): 2 \dot{b}^{\prime}+4 \dot{c}^{\prime}+b^{\prime}\left(\dot{b}+\frac{2}{\tau}\right)+2 \dot{c} c^{\prime}-a^{\prime}\left(\dot{b}+2 \dot{c}+\frac{2}{\tau}\right)=0 \\
& (z z): a^{\prime \prime}+b^{\prime \prime}+2 c^{\prime \prime}-\frac{1}{z}\left(a^{\prime}+b^{\prime}+2 c^{\prime}\right)+\frac{1}{2}\left(a^{\prime 2}+b^{\prime 2}+2 c^{\prime 2}\right)=0 .
\end{aligned}
$$

In the above expressions the first parenthesis is for the corresponding metric component, the dot for the proper-time $\tau$-derivative and the prime for the $z$-derivative.

Expanding the $(z z)$ component of Einstein's equations as a power series in $z$, and keeping only the leading terms for $z \rightarrow 0$, one obtains the tracelessness condition $-\epsilon(\tau)+$

$p_{\|}(\tau)+2 p_{\perp}(\tau)=0$ of $\left\langle T_{\mu \nu}\right\rangle$. The $(z z)$ equation thus encodes holographically the scale invariance of the boundary gauge theory. Moreover, that Einstein's equation does not 
features any $\tau$-derivative. Hence, at each proper time, if the $z$-dependence of two of the functions $a, b$ and $c$ are given, the third one is completely determined by the $(z z)$ equation and the requirement of a $z^{4}$ behavior near the boundary.

However, for completeness, let us discuss also some of the other Einstein's equations. Expanding the $(z \tau)$ component of Einstein's equations as a power series in $z$, one finds that the leading terms near the boundary give the relation $\dot{p}_{\|}+2 \dot{p}_{\perp}+\left(\epsilon+p_{\|}\right) / \tau=0$ which, assuming $\left\langle T_{\mu}^{\mu}\right\rangle=0$, is equivalent to the energy conservation equation in the CFT. Hence, the $(z \tau)$ and $(z z)$ components of Einstein's equations together encodes holographically both energy conservation and conformal invariance in the CFT.

One should remark that we have five equations $(4.10)$ for only three unknown functions. There is indeed some redundancy in Einstein's equations (4.10). It is e.g. consistent to replace the first three equations $(\tau \tau),(y y)$ and $(\perp \perp)$ by the combination $-(\tau \tau)+(y y)+$ $2(\perp \perp)-e^{a}(z z)$, which writes

$$
\dot{c}\left(\dot{c}+2 \dot{b}+\frac{4}{\tau}\right)=e^{a}\left\{2 b^{\prime \prime}+4 c^{\prime \prime}+b^{2}+3 c^{2}+2 b^{\prime} c^{\prime}-\frac{6}{z} b^{\prime}-\frac{12}{z} c^{\prime}\right\} .
$$

Hence, the system of Einstein's equations is only of order one in $\tau$. The required initial conditions should thus be two of the three functions $a_{0}(z), b_{0}(z)$ and $c_{0}(z)$, where $a_{0}=$ $a_{0}(z)=a(\tau=0, z)$ and analogous expressions for the others, because we have already at $\tau=0$ the constraint equation

$$
a_{0}^{\prime \prime}+b_{0}^{\prime \prime}+2 c_{0}^{\prime}+\frac{1}{2}\left(a_{0}^{\prime}\right)^{2}+\frac{1}{2}\left(b_{0}^{\prime}\right)^{2}+\left(c_{0}^{\prime}\right)^{2}-\frac{1}{z}\left(a_{0}^{\prime}+b_{0}^{\prime}+2 c_{0}^{\prime}\right)=0,
$$

We shall see in the next section that requiring a regular behavior of the solutions in the limit $\tau \rightarrow 0$ further reduces the freedom for the initial condition to only one arbitrary function of $z$.

The nonlinear character of equation (4.12) turns out to play a crucial role in the whole analysis. Naively, one would expect to be able to consider a small fluctuation over empty $A d S_{5}$ in the linearized approximation neglecting the quadratic terms in the above constraint. However it turns out, as will be shown below, that even if starting from e.g. an infinitesimal $a$, the nonlinear equation for $c$ will always generate a singularity for some large but finite $z$ ! Thus linearized fluctuations cannot be used as initial conditions in the boost-invariant kinematics. Moreover, a singularity, presumably related to an horizon, has to be present from the outset already in the initial conditions. This point will be addressed later in the paper.

To simplify 4.12) it is useful to introduce the following notation

$$
\begin{aligned}
v\left(z^{2}\right) & =\frac{1}{4 z} a_{0}^{\prime}(z), \\
\varsigma\left(z^{2}\right) & =\frac{1}{4 z} b_{0}^{\prime}(z), \\
w\left(z^{2}\right) & =\frac{1}{4 z} c_{0}^{\prime}(z) .
\end{aligned}
$$

If the constraint equation has a regular solution, the derivatives $a_{0}^{\prime}, b_{0}^{\prime}$ and $c_{0}^{\prime}$ are bounded in the bulk and $v, \varsigma$ and $w$ vanish when $z \rightarrow \infty$. The constraint equation simplifies when 
written in terms of $v, \varsigma$ and $w$

$$
v^{\prime}+\varsigma^{\prime}+2 w^{\prime}+v^{2}+\varsigma^{2}+2 w^{2}=0
$$

where the prime now means the $z^{2}$-derivative. Integrating the above equation assuming the regularity conditions from $z=0$ to $\infty$ gives

$$
\int_{0}^{\infty}\left(v^{\prime}+\varsigma^{\prime}+2 w^{\prime}\right) d z^{2}+\int_{0}^{\infty}\left(v^{2}+\varsigma^{2}+2 w^{2}\right) d z^{2}=0
$$

The first integral vanishes due to the imposed boundary conditions leading to

$$
\int_{0}^{\infty}\left(v^{2}+\varsigma^{2}+2 w^{2}\right) d z^{2}=0 .
$$

This equality however is satisfied only for $v=\varsigma=w=0$, thus the only regular solution is trivial - the vacuum $A d S_{5}$. Therefore, at any time the metrics of interest must have a singularity at some value of $z$. In particular this will be the case even at $\tau=0$, with the meaning that any nontrivial initial condition consistent with the Einstein's equations will lead to a metric singularity at some value of $z$. The nonsingularity constraint on the geometry requires that all the singularities apart from the one sitting at $z=\infty$ will only be of coordinate nature. This provides a strong selection mechanism for the allowed initial conditions.

\subsection{Constraints at early time}

This section analyzes the constraints on initial conditions imposed by the Einstein's equations together with the assumption of nonsingularity of the geometry at $\tau=0$. The $(\tau z)$ component equation (see (4.10) ) takes the form

$$
\partial_{z} a-\partial_{z} b=\tau \cdot(\ldots)
$$

which means that at $\tau=0, a(\tau, z)$ and $b(\tau, z)$ can differ only by a constant. Since both functions have to vanish at $z=0$, this constant vanishes as well. Therefore

$$
a(0, z)=b(0, z) .
$$

Incidentally the condition (4.18) ensures also that the geometry is nonsingular on the light-cone $\tau=0$ for generic $z$.

In fact the constraint equation can be solved exactly leading to the space of solutions parametrized fully by the single function. The trick is to introduce the linear combinations

$$
\begin{aligned}
& v_{+}=-w-v \\
& v_{-}=w-v
\end{aligned}
$$

for which the equation (4.14) becomes algebraic for $v_{-}$(both $v_{-}$and $v_{+}$are understood as functions of $z^{2}$ ). After trivial algebra one obtains

$$
v_{-}=\sqrt{2 v_{+}^{\prime}-v_{+}^{2}} .
$$


Therefore all solutions of the initial value nonlinear constraint equations are parametrized by an arbitrary function $v_{+}\left(z^{2}\right)$. The next step is to analyze what further conditions must be imposed on $v_{+}\left(z^{2}\right)$. First, since the the metric coefficient functions have to vanish as $z^{4}$ one gets

$$
v_{+}\left(z^{2}\right) \sim \frac{2}{3} \varepsilon_{0} z^{6} \quad \text { for } \quad z \sim 0
$$

Moreover, from the arguments in section 4.2 it follows that there is a singularity at some finite $z=z_{0}$. The behavior at this singularity is constrained by demanding that this would be just a coordinate singularity and not a curvature blow-up. Assuming a power-like blowup of $v_{+}\left(z^{2}\right)$ at $z=z_{0}$, the regularity of the square of the Riemann tensor leads to the conclusion, that $v_{+}\left(z^{2}\right)$ has to have a first order pole

$$
v_{+}\left(z^{2}\right) \sim \frac{1}{z_{0}^{2}-z^{2}} \quad \text { for } \quad z \sim z_{0}
$$

with residue 1.

The coordinate singularity in $v_{+}$at $z=z_{0}$ translates directly into the behavior of the metric coefficients around $z_{0}$. This means that the proper time metric component has a second order zero at $z_{0}$ so that the metric at $\tau=0$ looks like

$$
d s^{2}=-\frac{1}{z^{2}}\left(1-\frac{z}{z_{0}}\right)^{2}\left\{d \tau^{2}+\tau^{2} d y^{2}\right\}+\ldots+\frac{1}{z^{2}} d z^{2}
$$

in the vicinity of $z=z_{0}$, reminiscent of the behavior of a horizon in Fefferman-Graham coordinates. Note, however, that at $\tau=0$ the term in curly braces becomes $d x^{+} d x^{-}$ in contrast to a 'Schwarzschild' horizon where the corresponding structure is of the form $-\left(1-z / z_{0}\right)^{2} d t^{2}+\ldots$. Presumably the metric singularity could be avoided by using a different set of coordinates (e.g. Eddington-Finkelstein ones). Then it would be also possible to apply the framework of dynamical horizons to check and locate eventual trapped surfaces. However performing a change of variables from Fefferman-Graham to EddingtonFinkelstein ones is a formidable task, since contrary to late proper-time regimes, $\tau \sim 0$ Fefferman-Graham is not mapped in the bulk into $\tilde{\tau} \sim 0$ Eddington-Finkelstein. This means that the change of variables cannot be performed perturbatively and is beyond the scope of analytical methods presented here. It would be however interesting to analyze in detail the local structure of the geometry at $z=z_{0}$ including time-dependence. This problem is left for future work.

It is interesting for further discussion to present explicit solutions of the constraints. One of them arises from the choice

$$
v_{+}=a\left(\tan a z^{2}-\tanh a z^{2}\right) .
$$

The initial metric profiles may be integrated explicitly to obtain

$$
\begin{aligned}
& a_{0}(z)=2 \log \cos a z^{2}, \\
& c_{0}(z)=2 \log \cosh a z^{2} .
\end{aligned}
$$


The above solution possesses a (coordinate - not leading to any curvature singularities) singularity at

$$
z_{0}=\sqrt{\frac{\pi}{2 a}}
$$

More generally one can parametrize $v_{+}$in the following manner

$$
v_{+}\left(z^{2}\right)=\frac{2}{3} \epsilon_{0} z_{0}^{2} \cdot \frac{z^{6}}{z_{0}^{2}-z^{2}} f\left(z^{2}\right),
$$

where $f(0)=1, f\left(z_{0}^{2}\right)=\frac{3}{2 \epsilon_{0} z_{0}^{8}}$ and otherwise is a regular function of $z^{2}$ variable for $z<\infty$. One has also to ascertain that $v_{-}$obtained from (4.20), does not become complex

Once the allowed initial conditions are under control one may proceed to find the solution of Einstein's equations starting with these initial data.

\subsection{Early time expansion of the energy density}

The Einstein's equations (4.9) can be solved for any energy density perturbatively in $z^{2}$. Starting with some arbitrary energy density $\epsilon$, the first three nontrivial terms in the expansion of $a(\tau, z)$ warp factor take the form

$$
\begin{aligned}
a(\tau, z)= & -\epsilon(\tau) \cdot z^{4}+\left\{-\frac{\epsilon^{\prime}(\tau)}{4 \tau}-\frac{\epsilon^{\prime \prime}(\tau)}{12}\right\} \cdot z^{6}+\left\{\frac{1}{6} \epsilon(\tau)^{2}+\frac{1}{6} \tau \epsilon^{\prime}(\tau) \epsilon(\tau)+\frac{1}{16} \tau^{2} \epsilon^{\prime}(\tau)^{2}\right. \\
& \left.+\frac{\epsilon^{\prime}(\tau)}{128 \tau^{3}}-\frac{\epsilon^{\prime \prime}(\tau)}{128 \tau^{2}}-\frac{\epsilon^{(3)}(\tau)}{64 \tau}-\frac{1}{384} \epsilon^{(4)}(\tau)\right\} \cdot z^{8}+\cdots
\end{aligned}
$$

This power series can be extended to an arbitrary order in $z^{2}$ by solving Einstein's equations and the only obstructions are of a purely computational nature. Generically terms in the expansion (4.28) contain inverse powers of proper time multiplying the energy density and its derivatives. Assuming the energy density can be expanded in a regular power series around $\tau=0$, the singular inverse powers of proper time in (4.28) will be present unless all the odd terms vanish. This requirement constrains the energy density in the early times domain to be a power series in even powers of $\tau$, namely

$$
\epsilon(\tau)=\epsilon_{0}+\epsilon_{2} \tau^{2}+\epsilon_{4} \tau^{4}+\ldots .
$$

On the other hand, taking $\tau$ to be zero in equation (4.28) gives the relation between the early times energy density and the profile of $a_{0}(z)$, which has been signaled in subsection (4.1). Basically expanding the initial profile of the metric in the radial AdS variable near the boundary and comparing with (4.28) at $\tau=0$

$$
a(0, z)=a_{0}(z)=-\epsilon_{0} \cdot z^{4}-\frac{2}{3} \epsilon_{2} \cdot z^{6}+\left(-\frac{\epsilon_{4}}{2}-\frac{\epsilon_{0}^{2}}{6}\right) \cdot z^{8}+\ldots
$$

allows one to solve for all the $\epsilon_{2 i}$ sitting in (4.29). This pattern continues to any order in $z$ (and thus $\tau$ ) expansion. Note also, that each parameter in the expansion of energy density around $\tau=0$ is an independent dimensionful quantity and in order to fully specify 
the initial conditions infinitely many such terms are needed. This is in stark contrast to the late time behavior, where only one dimensionful constant $(\Lambda)$ appears. Physically, this is in agreement with the fact that in the thermally equilibrated final stages of Bjorken expansion all differences due to initial data should have been washed away by dissipative effects and the only parameter characterizing the flow is an overall energy scale (given e.g. as the energy density at a certain fixed proper time $\tau_{0}$ ).

The strategy for finding the time evolution from given initial data is to use the Einstein's equations to generate the expansion of the metric warp-factors for sufficiently high order (the bigger, the better, but for technical reasons this has been achieved up to the order $z^{84}$ or equivalently $\tau^{80}$ in most cases) and then for given regular (with finite curvature evaluated on initial data for $z<\infty$ ) initial profile generate the power series for the energy density at early times. Thus the AdS/CFT correspondence beautifully sets the allowed initial conditions for gauge theory dynamics using the gravitational description. However since the series (4.29) has generically a finite radius of convergence, some resummation method is needed in order to extend it to larger proper times.

\section{Transition to the hydrodynamic regime}

\subsection{Resummation scheme for the energy density}

It is desirable now to find a suitable numerical approach, relating the solutions of the Einstein's equations (4.10) to the physical quantities, energy density and pressures, as a function of $\tau$.

Though early time dynamics of the field theory is dictated by the initial conditions, after certain time the system is expected to settle down to local equilibrium. For $\tau$ sufficiently large the plasma should exhibit the universal hydrodynamic behavior, where the only trace of the initial conditions is given by the overall scale $\Lambda$, see (3.13). In order to track the dynamics of the system with sufficient accuracy from $\tau=0$ to $\tau \gg 1$, numerical methods are needed. In particular the early-times power series for the energy density has a finite radius of convergence and a resummation is needed in order to find its behavior for large $\tau$.

We would like to address the following questions. Firstly, whether during the evolution from some (generic) initial data at $\tau=0$ one can observe a passage to the asymptotic perfect fluid behaviour

$$
\epsilon(\tau) \sim \frac{1}{\tau^{4 / 3}}+\ldots
$$

To be more general one may try to determine the asymptotic exponent $s$ in

$$
\epsilon(\tau) \sim \frac{1}{\tau^{s}}+\ldots
$$

and determine whether it is significantly different (as is expected) from the free streaming value $s=1$. 


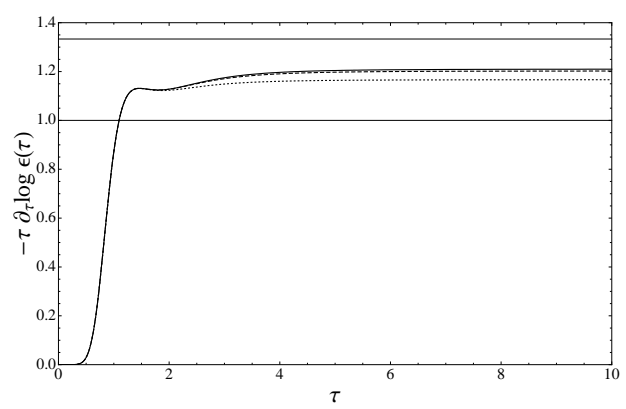

Figure 2: Approximate value of $s$ obtained from the logarithmic derivative and Pade approximation for A) $N_{c u t}=32$ (dotted line), B) $N_{c u t}=40$ (dashed line) and C) $N_{c u t}=48$ (solid line) for initial profile $v_{+}^{(1)}\left(z^{2}\right)$. Two horizontal lines denote $s=1$ (free streaming scenario) and $s=4 / 3$ (perfect fluid case).

As explained above, it is quite difficult to answer this question as the energy density in the early time regime takes the form

$$
\epsilon(\tau)=\epsilon_{0}+\epsilon_{2} \tau^{2}+\ldots+\epsilon_{2 N_{c u t}} \tau^{2 N_{c u t}}+\ldots
$$

where $N_{\text {cut }}$ is a natural number denoting the cut-off up to which the evaluation of the energy density from the initial profile in the bulk has been performed. In most cases $N_{c u t}=40$ and increasing this accuracy is difficult. Moreover the series has a finite range of convergence. In order to estimate the asymptotic exponent $s$ appearing in (5.2) it is convenient to express it through a logarithmic derivative

$$
s=-\lim _{\tau \rightarrow \infty} \tau \cdot \frac{\mathrm{d}}{\mathrm{d} \tau} \log \epsilon(\tau)
$$

and perform a Pade approximation (of order $\left(N_{\text {cut }}, N_{\text {cut }}\right)$ ) to the r.h.s. of (5.4) with $\varepsilon(\tau)$ substituted with our power series (5.3):

$$
s_{\text {approx }}=\frac{s_{U}^{(0)}+s_{U}^{(2)} \tau^{2}+\ldots+s_{U}^{\left(2 N_{c u t}\right)} \tau^{2 N_{c u t}}}{s_{D}^{(0)}+s_{D}^{(2)} \tau^{2}+\ldots+s_{D}^{\left(2 N_{c u t}\right)} \tau^{2 N_{c u t}}} .
$$

$s=4 / 3$ corresponds then to the perfect fluid case and $s=1$ to the free-streaming scenario. Of course it is not expected that (5.5) will give $s=4 / 3$ exactly ${ }^{12}$.

Let us consider the three following examples of the initial profile, satisfying the curvature nonsingularity constraint, the first one being (4.24), the second its deformed variant, and the third one from the family (4.27):

$$
\begin{aligned}
v_{+}^{(1)}\left(z^{2}\right) & =\tan \left(z^{2}\right)-\tanh \left(z^{2}\right), \\
v_{+}^{(2)} & =\tan \left(z^{2}\right)-\tanh \left(z^{2}+\frac{z^{8}}{6}\right), \\
v_{+}^{(3)}\left(z^{2}\right) & =\frac{2}{3} \frac{z^{6}}{1-z^{2}}\left(1+\frac{1}{2} z^{2}\right) .
\end{aligned}
$$

\footnotetext{
${ }^{12}$ Such a Pade approximation has, by construction, a different subleading large $\tau$ behaviour from viscous hydrodynamics.
} 
The obtained values of $s$ for the first profile, for which we had a power series in $\tau$ up to order $\tau^{100}$, are $s_{\text {approx }}=1.1667,1.1923,1.2025,1.2025$ and 1.2101 respectively for $N_{\text {cut }}=32,36,40,44$ and 48 . The approximate value of $s$ is closer to $s=4 / 3$ than to $s=1$ for largest $N_{c u t}$. For the second and third profile the results are inconclusive, since the corresponding energy densities are provided with worse accuracy.

In the rest of the text, we shall keep the asymptotic perfect fluid value $s=4 / 3$. Then, a suitable Pade resummation scheme, with the required asymptotia can be given by

$$
\epsilon_{\text {approx }}^{3}(\tau)=\frac{\epsilon_{U}^{(0)}+\epsilon_{U}^{(2)} \tau^{2}+\ldots+\epsilon_{U}^{\left(N_{c u t}-2\right)} \tau^{N_{c u t}-2}}{\epsilon_{D}^{(0)}+\epsilon_{D}^{(2)} \tau^{2}+\ldots+\epsilon_{D}^{\left(N_{c u t}-2\right)} \tau^{N_{c u t}+2}} .
$$

where both $\epsilon_{D}^{2 i}$ and $\epsilon_{U}^{2 i}$ are obtained by expanding (5.7) around $\tau=0$ and comparing with (5.3). Such a resummation imposes the correct asymptotic behavior, but differs, by construction, in the subleading behavior with viscous hydrodynamics.

$$
\epsilon_{\text {approx }}(\tau)=\frac{1}{\tau^{4 / 3}}\left\{\#+\frac{1}{\tau^{2}} \cdot \#\right\},
$$

(the first subleading piece scales as $\tau^{-2}$ whereas the correct scaling is $\tau^{-2 / 3}$ ). This difference is not substantial and can be cured by more refined resummation schemes ${ }^{13}$.

Despite its simplicity, it turns out that the resummation (5.7) works pretty well (the results seems to converge well with increasing cut-off, see Figs.2 and 4 in extending the energy density beyond the convergence radius of the early-time power series (which is its main task) and in providing a qualitative picture of dynamics.

\subsection{Qualitative features of the approach to local equilibrium}

The method (5.7) can be used to study the approximate behavior of the energy density as a function of time for large enough times to see local equilibration. Fig.3 shows the plots of energy density as a function of proper time for the three profiles (5.6) obtained for the highest cut-offs. The results seems to converge well for the first profile, see Fig.4. Energy densities obtained for these profiles differ at the initial stages, whereas in the late-time regimes both seem to approach local equilibrium. A measure of the local equilibrium is the relative difference between the transverse and perpendicular pressures defined as

$$
\Delta p(\tau)=1-\frac{p_{\|}(\tau)}{p_{\perp}(\tau)}
$$

When this quantity is close to zero, it signals the isotropisation indicating local equilibrium, while a value of order one is an indication in favor of the free streaming scenario. Fig 5 shows the plot of the relative difference of pressures (5.9). It is interesting to note the rapid fall-off of the pressure difference on a scale $\tau=\mathcal{O}(1)$. This fall-off appears to be stable after different numerical checks. Interestingly enough, there is a bump which prevents the pressure to reach isotropy before $\tau=\mathcal{O}(5)$. However, the Pade approximants

\footnotetext{
${ }^{13} \mathrm{An}$ other possible issue are roots of the denominator lying within the range $(0, \infty)$. If such feature is encountered, it can be interpreted as an artifact of approximation without real physical significance.
} 


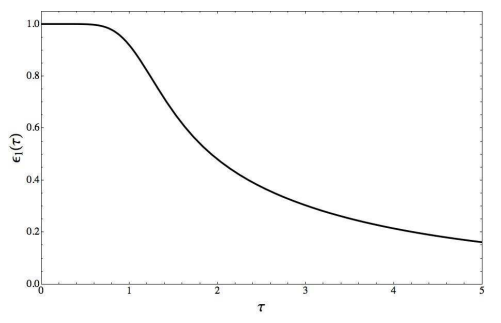

A)

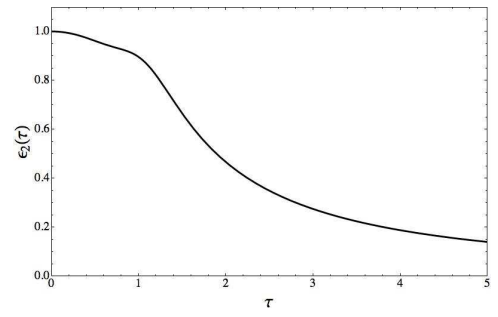

$B)$

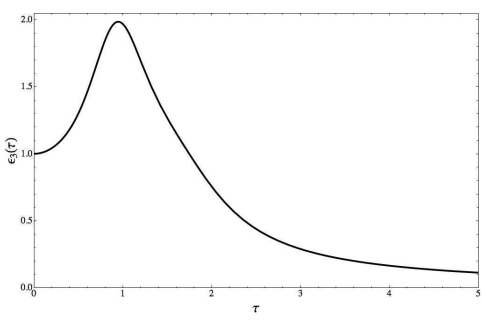

$C)$

Figure 3: A) Energy density $\epsilon_{1}(\tau)$ as a function of proper-time $\tau$ obtained from Pade approximation for cut-off $N_{c u t}=46$ and initial profile $v_{+}^{(1)}\left(z^{2}\right)$ in the bulk; B) Energy density $\epsilon_{2}(\tau)$ for the second profile. C) Energy density $\epsilon_{3}(\tau)$ as a function of proper-time $\tau$ obtained from Pade approximation for cut-off $N_{c u t}=34$ and initial profile $v_{+}^{(3)}\left(z^{2}\right)$ in the bulk.

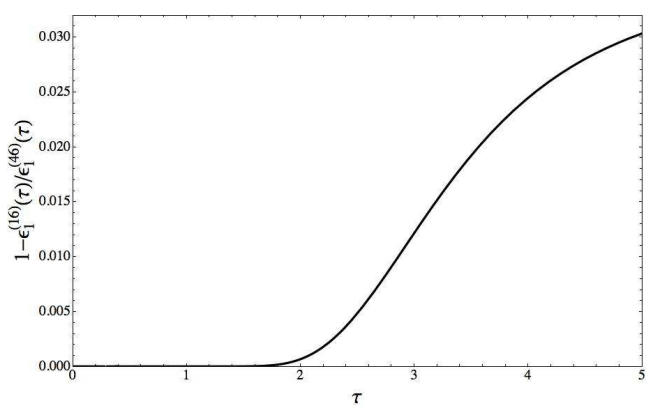

Figure 4: Relative difference between the energy densities for the first profile for cut-offs $N_{c u t}=16$ and $N_{c u t}=46$ does not exceed $10 \%$.

for the pressure difference are less stable than for the energy density and the differences appear after the bump. The second profile which is a slight deformation of the first one does not seem to exhibit this bump. In any case, it would be physically interesting to check whether this phenomenon of rapid fall-off but incomplete isotropization is or not a characteristic feature of the strong coupling evolution. We intend to analyze this issue using numerical methods for solving Einstein's equations in future work.

\section{Summary}

This paper studies the early-times dynamics of boost-invariant plasma using analytical methods. The motivations for this work are both phenomenological and theoretical. On the one hand, the recent findings of the RHIC experiment suggested that locally equilibrated nuclear matter behaves as an almost perfect fluid, which presumably indicates a strongly coupled regime (at least at this stage of its evolution) of the underlying gauge field theory, i.e. QCD. Despite the fact that realistic dynamics of the QCD after the collision would require the understanding of rapidity dependence (i.e. deviations from boost-invariant dynamics, which is only approximately valid in the central rapidity region) and might be 

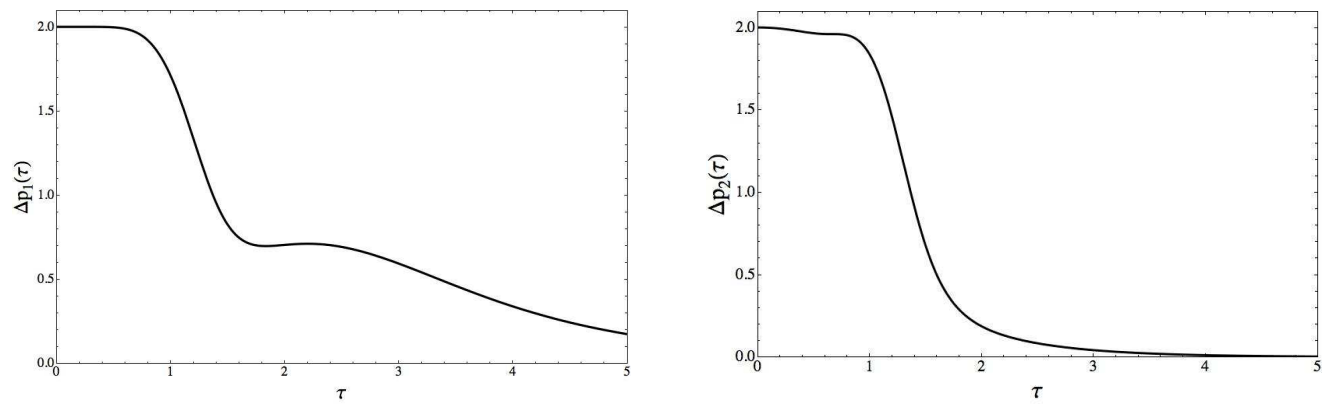

Figure 5: Relative difference in pressures for the first (left) and second (right) profiles - for $\tau \approx 1$ there is a rapid fall-off but (perhaps, see text) does not reach yet a complete izotropization for the first profile.

driven as well by the perturbative effects (as for the Color Glass Condensate [9] initial conditions), it is still interesting to consider the boost-invariant expansion of a conformal plasma at strong coupling as a useful toy-model for e.g. estimates of the thermalization time. On the other hand, theoretically, the boost-invariant plasma evolution is a workable example of a dynamical system at strong coupling, which is interesting on its own. The modern developments translates (using the AdS/CFT correspondence) the dynamics of the strongly coupled gauge theories into the evolution of higher dimensional space-time equipped with a nontrivial metric. Thus, as suggested previously by various authors (e.g. [30]) the thermalization of the excited gauge theory matter should be dual to black hole (or black brane) formation (see [31, 32] for concrete realizations of this observation), which is obtained in late time as the dual of the Bjorken hydrodynamical flow. This subject is very fresh and thus any work which may shed light on this fascinating process is valuable.

The main result of the studies presented here is that the boost-invariant dynamics of a strongly coupled conformal plasma is sensitive to the initial conditions. This contradicts the scaling hypothesis [23], which, analogous to the late-time case [7] would indicate some uniqueness of the early-times solution. In fact the scaling does not occur due to a subtlety - the a priori dominant scaling contributions vanish precisely in the limit $s \rightarrow 0$. (see subsection (4.1) for details). The correct physical picture leads to a link between the early-time expansion of the energy density and the initial profile of the bulk metric. We find a quite general result that at all times, including the initial one, a singularity of at least one metric coefficient should develop in the bulk. Hence, the requirement that this does not lead to real curvature singularities already at initial time is a basic constraint on the possible initial conditions. The analysis of the possible curvature singularities in the initial data at finite distance from the boundary fixed the early times power series for the energy density to contain only even powers of proper time. We have shown that solving the nonlinear constraint equation in the Fefferman - Graham coordinates leads to the conclusion that the initial data must contain a coordinate singularity in the bulk of AdS which may signal the presence of a dynamical horizon (we are making a distinction w.r.t. the event horizon which is a global notion) right from the start of the evolution. 
This suggests that the process of thermalization in this boost-invariant setting does not amount to a formation of a black hole, as was suggested up till now, but rather amounts to a qualitative difference in the behaviour of the horizon. We plan to investigate these issues in more detail in future work using numerical methods.

Interesting further directions of study include numerical investigation of the bulk evolution dual to the boost-invariant flow. This can be achieved using the methods presented in [31] and may provide precise numerical results covering both early, intermediate and late time regimes (see 33] for an interesting study of boost invariant flow sourced by boundary metric perturbations). It is important to understand more qualitatively the relation between the initial conditions in the bulk and the shape of the energy density as function of proper time, which would give the more precise estimates on the thermalization time. Moreover the methods developed in this paper are well suited to reconsider the problem of plasma isotropisation posed in [25] using analytical methods (note that anisotropic energymomentum tensor should reach the equilibrium exponentially fast, whereas hydrodynamic evolution leaves a power-like tail). Finally the most interesting, yet highly non-trivial, extensions of the AdS/CFT program for the dynamical evolution of a plasma are the studies of the initial conditions such as shock waves collision using the dual gravity picture (see for instance [34, 35] for some preliminary attempts). We hope that the general properties and their numerical implementation we found can serve as a testing ground for the investigation on the proper initial conditions and evolution of the plasma. The authors plan to address some of the mentioned issues in the nearest future.

\section{Acknowledgments}

The authors would like to thank Ofer Aharony and Paul Chesler for discussions. During the course of this work, MH has been supported by Polish/French Research Programme Polonium, Polish Ministry of Science and Information Technologies grant N N202 247135 (2008-2010), Foundation for Polish Science award START and Helena Kogutowska Scientific Scholarship by Jagiellonian University. MH and RJ would like to thank the Institute for Theortical Physics of Saclay for hospitality. MH and RJ were supported by Polish science funds during 2009-2011 as a research project (NN202 105136). The participation of RP and RJ in this investigation was partly supported by 6 Program of European Union "Marie Curie Transfer of Knowledge" Project: Correlations in Complex Systems "COCOS" MTKD-CT-2004-517186. R.P. wants to thank the Insitute of physics of the Jagiellonian university in Cracow for hospitality.

\section{References}

[1] E. V. Shuryak, "What RHIC experiments and theory tell us about properties of quark-gluon plasma?," Nucl. Phys. A 750, 64 (2005).

[2] P. F. Kolb and U. W. Heinz, "Hydrodynamic description of ultrarelativistic heavy-ion collisions," [arXiv: nucl-th/0305084;

P. Huovinen and P. V. Ruuskanen, "Hydrodynamic models for heavy ion collisions," [arXiv: nucl-th/0605008. 
[3] J. Y. Ollitrault, "Anisotropy As A Signature Of Transverse Collective Flow," Phys. Rev. D 46, 229 (1992);

See also the recent reviews: J. Y. Ollitrault, "Relativistic hydrodynamics," Eur. J. Phys. 29, 275 (2008);

T. Hirano, N. van der Kolk and A. Bilandzic, "Hydrodynamic and Flow".

[4] J. M. Maldacena, "The large N limit of superconformal field theories and supergravity," Adv. Theor. Math. Phys. 2, 231 (1998) [Int. J. Theor. Phys. 38, 1113 (1999)];

S. S. Gubser, I. R. Klebanov and A. M. Polyakov, "Gauge theory correlators from non-critical string theory," Phys. Lett. B 428, 105 (1998);

E. Witten, "Anti-de Sitter space and holography," Adv. Theor. Math. Phys. 2, 253 (1998).

[5] G. Policastro, D. T. Son and A. O. Starinets, "The shear viscosity of strongly coupled N = 4 supersymmetric Yang-Mills plasma," Phys. Rev. Lett. 87, 081601 (2001);

P. Kovtun, D. T. Son and A. O. Starinets, "Viscosity in strongly interacting quantum field theories from black hole physics," Phys. Rev. Lett. 94, 111601 (2005).

[6] J. D. Bjorken, "Highly Relativistic Nucleus-Nucleus Collisions: The Central Rapidity Region," Phys. Rev. D 27, 140 (1983).

[7] R. A. Janik and R. B. Peschanski, "Asymptotic perfect fluid dynamics as a consequence of AdS/CFT," Phys. Rev. D 73, 045013 (2006),

"Gauge / gravity duality and thermalization of a boost-invariant perfect fluid," Phys. Rev. D 74, 046007 (2006).

[8] S. Bhattacharyya, V. E. Hubeny, S. Minwalla and M. Rangamani, "Nonlinear Fluid Dynamics from Gravity," JHEP 0802, 045 (2008).

[9] For a review: E. Iancu and R. Venugopalan, "The color glass condensate and high energy scattering in QCD," arXiv:hep-ph/0303204.

[10] G. Beuf, "Gravity dual of N=4 SYM theory with fast moving sources," arXiv:0903.1047 [hep-th].

[11] R. A. Janik, "Viscous plasma evolution from gravity using AdS/CFT," Phys. Rev. Lett. 98, $022302(2007)$.

[12] M. P. Heller and R. A. Janik, "Viscous hydrodynamics relaxation time from AdS/CFT," Phys. Rev. D 76, 025027 (2007).

[13] H. Epstein, V. Glaser and A. Jaffe, "Nonpositivity of energy density in Quantized field theories," Nuovo Cim. 36, 1016 (1965), quoted in Ref. 34.

[14] C. Fefferman and C.R. Graham, "Conformal Invariants," in Elie Cartan et les Mathématiques d'aujourd'hui, Astérisque (1985) 95.

[15] S. de Haro, S. N. Solodukhin and K. Skenderis, "Holographic reconstruction of spacetime and renormalization in the AdS/CFT correspondence," Commun. Math. Phys. 217, 595 (2001) [arXiv: hep-th/0002230];

K. Skenderis, "Lecture notes on holographic renormalization," Class. Quant. Grav. 19, 5849 (2002) [arXiv: hep-th/0209067].

[16] S. Nakamura and S. J. Sin, "A holographic dual of hydrodynamics," JHEP 0609, 020 (2006).

[17] D. Bak and R. A. Janik, "From static to evolving geometries: R-charged hydrodynamics from supergravity," Phys. Lett. B 645, 303 (2007). 
[18] P. Benincasa, A. Buchel, M. P. Heller and R. A. Janik, "On the supergravity description of boost invariant conformal plasma at strong coupling," Phys. Rev. D 77, 046006 (2008).

[19] A. Buchel, "On SUGRA description of boost-invariant conformal plasma at strong coupling," AIP Conf. Proc. 1031, 196 (2008) [arXiv:0803.3421 [hep-th]].

[20] M. P. Heller, P. Surowka, R. Loganayagam, M. Spalinski and S. E. Vazquez, "On a consistent AdS/CFT description of boost-invariant plasma," Phys. Rev. Lett. 102, 041601 (2009), arXiv:0805.3774 [hep-th].

[21] S. Kinoshita, S. Mukohyama, S. Nakamura and K. y. Oda, "A Holographic Dual of Bjorken Flow," arXiv:0807.3797 [hep-th].

[22] M. P. Heller, R. A. Janik and R. Peschanski, "Hydrodynamic Flow of the Quark-Gluon Plasma and Gauge/Gravity Correspondence," Acta Phys. Polon. B 39, 3183 (2008) [arXiv:0811.3113 [hep-th]].

[23] Y. V. Kovchegov and A. Taliotis, "Early time dynamics in heavy ion collisions from AdS/CFT correspondence," Phys. Rev. C 76, 014905 (2007).

[24] P. Figueras, V. E. Hubeny, M. Rangamani and S. F. Ross, "Dynamical black holes and expanding plasmas," arXiv:0902.4696 [hep-th].

[25] R. A. Janik and P. Witaszczyk, "Towards the description of anisotropic plasma at strong coupling," JHEP 0809, 026 (2008).

[26] A. Buchel, "Finite temperature resolution of the Klebanov-Tseytlin singularity," Nucl. Phys. B 600, 219 (2001) [arXiv:hep-th/0011146].

[27] R. Baier, P. Romatschke, D. T. Son, A. O. Starinets and M. A. Stephanov, "Relativistic viscous hydrodynamics, conformal invariance, and holography," JHEP 0804, 100 (2008).

[28] I. Booth, M. P. Heller and M. Spalinski, "Black brane entropy and hydrodynamics: the boost-invariant case," arXiv:0910.0748 [hep-th].

[29] I. Booth, "Black hole boundaries," Can. J. Phys. 83, 1073 (2005) [arXiv:gr-qc/0508107].

[30] H. Nastase, "The RHIC fireball as a dual black hole," arXiv:hep-th/0501068.

[31] P. M. Chesler and L. G. Yaffe, "Horizon formation and far-from-equilibrium isotropization in supersymmetric Yang-Mills plasma," arXiv:0812.2053 [hep-th].

[32] S. Bhattacharyya and S. Minwalla, "Weak Field Black Hole Formation in Asymptotically AdS Spacetimes," JHEP 0909, 034 (2009) [arXiv:0904.0464 [hep-th]].

[33] P. M. Chesler and L. G. Yaffe, "Boost invariant flow, black hole formation, and far-from-equilibrium dynamics in $\mathcal{N}=\mathbf{4}$ supersymmetric Yang-Mills theory," arXiv:0906.4426 [hep-th].

[34] D. Grumiller and P. Romatschke, "On the collision of two shock waves in AdS5," JHEP 0808, 027 (2008).

[35] J. L. Albacete, Y. V. Kovchegov and A. Taliotis, "Modeling Heavy Ion Collisions in AdS/CFT," JHEP 0807, 100 (2008). 